\title{
Using the Biological Feedback Method in Speech Therapy for the Formation of Speech Breathing in Preschool Children with Erased Dysarthria
}

Olga Michailovna Shterts ${ }^{\dagger}$

\section{Abstract}

The article discusses the problem of the formation of speech breathing in preschool children with an erased form of dysarthria using the biological feedback method. In children suffering from speech impairment, the work of the respiratory system is not normal. Disorders of prosody supplement violations of the soundproducing side of speech. As a result, in children, including the ones with dysarthria, speech becomes unclear and slurred. The theoretical part of the study considers the specificity of the prosodic side of speech in children with an erased form of dysarthria from the point of view of various researchers. The problem of dependence between the prosodic side of speech and the severity of a speech defect is considered. The practical part of the study reveals the content of the organisation of the empirical study on the formation of diaphragmatic relaxation breathing using a hardware-diagnostic complex "BF" (Biological Feedback). It has been established that the biological feedback method contributes to the formation of diaphragmaticrelaxation breathing in preschool children with an erased form of dysarthria. In pre-schoolers, suffering from erased dysarthria and having a history of attention deficit hyperactivity syndrome, the process of formation of diaphragmatic relaxation breathing becomes more complicated.

Keywords: Erased Dysarthria; Prosodic Side of Speech; Speech Breathing; Diaphragmatic Relaxation Breathing; Biological Feedback Method; Preschool Children

\footnotetext{
${ }^{\dagger}$ Elabuga Institute of Kazan Federal University, Tatarstan 423600, g. Elabuga, str. Kazan, 89, Email: olgashterz@mail.ru (C) 2020 Shterts. This is an Open Access article distributed under the terms of the Creative Commons Attribution License (http://creativecommons.org/licenses/by/2.0), which permits unrestricted use, distribution, and reproduction in any medium, provided the original work is properly cited.
} 


\section{Introduction}

Breathing is the most critical life-support function because it carries out the gas exchange. The development of the respiratory function, both of a child without speech impairment, and, in particular, of a child with a speech impairment is necessary. Formed speech breathing physiologically ensures the smoothness of oral speech. This contributes to the clarity of presentation and a better understanding of speech by the interlocutor.

In children with speech impairment, physiological respiration has significant deviations from the standard, especially in children with dysarthria. This negatively affects the formation of their speech breathing; speech becomes not clear, slurred, which makes it more difficult for the interlocutor to understand the speech. Speech breathing does not develop spontaneously, without the use of targeted speech therapy technologies (Dinh, 2019; Kireev et al., 2019; Rupeika-Apoga et al., 2019; Pavlyshyn et al., 2019; Saenko et al., 2019; Shrestha, 2019; Tarman, 2016).

Therefore, it is crucial to prevent the violation of speech breathing in children with an erased form of dysarthria from preschool age until the incorrect stereotype of speech breathing is finally fixed.

Hitos et al. note that oral breathing can affect speech development, socialisation, and academic performance. Early detection of mouth breathing is vital to prevent and minimise its adverse effects on the overall development of people (Hitos et al., 2013).

The structure of speech therapy classes includes the work on the formation of speech breathing. A speech therapist forms speech breathing by using breathing exercises. However, this process takes a long time and does not always bring the proper result (Bernardi, 2019; Chistobaev et al., 2018). Therefore, it was important for the authors to determine how the hardwarediagnostic complex "BF", which is based on the biological feedback method, affects the formation of speech breathing in preschool children with an erased form of dysarthria. The study begins with a brief description of methodology. Following this, it discusses the research results, its organisation and findings.

\section{Methods}

As a methodological toolkit, the authors used a hardware-diagnostic complex "BF" (BF-Health Room, Logotherapeutic Room), a set of exercises for the formation of speech breathing proposed by Belyakova. Research results were processed using the method of mathematical statistics (Student's t-test).

A logotherapeutic method of biological feedback authored by Smetankin is a unique method for the formation of speech breathing and the normalisation of the functional state in oral speech for more effective correction of speech disorders of various origins (Smetankin, 2007). The uniqueness of this method lies in the possibility of teaching a child how to selfregulate his/her speech breathing.

This computer program, combined with a set of standard and variable games, turns the logotherapeutic procedure into an entertaining game, which corresponds to neoplasms of preschool age and the leading type of children's activity.

Changes in body processes (via sensors connected to the computer) are immediately displayed on the screen - the readings change, graphics change or music may be quieter or louder. In order to regulate, adjust the operation of certain systems or organs, a person must consciously use the biological feedback to change the information on the screen in the right direction (Etherington, 2019; Brigas, 2019) This means that a person can learn to adjust the body function with a little effort of will in such a way as to improve his/her health.

This technology can be used in the cases of stuttering, alalia, aphasia, dysgraphia and dyslexia, dyslalia, dysarthria, rhinolalia, phonation disorder due to paresis and paralysis of the larynx, functional dysphonia, impaired speech rate, so-called speech anxiety, speech 
disorders in children and adults with impaired hearing and vision.

Thirty-six children aged five with an erased form of dysarthria who attended a preschool educational institution took part in the empirical study conducted by the authors. It should be noted that the study sample included children who only attended speech therapy classes for 2 or 3 months. In total, 20 correctionaldevelopmental sessions on the formation of speech breathing were conducted.

\section{Research}

Erased dysarthria is characterised by the impairment of sound pronunciation and the prosodic side of speech, which is caused by micro neurological symptoms (Volkova, 2004). Darley defines dysarthria as a group of speech disorders resulting from disturbances in muscle tissue and control of the mechanism of speech production (Darley et al., 1969).

This disorder is based on slight residual disturbances in the innervation of the articulatory, voice and breathing apparatus, which are detected only with an in-depth neurological examination. In speech symptoms, in addition to impaired sound pronunciation and phonemic hearing, prosodic disorders are observed: speech is monotonous, unexpressive, the pitch of the voice is often low, the voice is quiet, the pace of speech is slowed or accelerated.

Russell J., in his book, examines the relationship of dysarthria with neurological disorders. The author identifies five types of dysarthria in children and explains the classification of dysarthria in children (Russell, 1999).

Henrike Blumenfeld thinks that the impairment of motor signals with the formation of speech leads to dysarthria (Blumenfeld, 2017). He proved that the damage to the lower motor neurons going from the spine to the muscles leads to flaccid dysarthria, which affects all aspects of speech activity: articulators, soft palate, larynx, and respiratory system. Flaccid dysarthria is often observed along with swallowing problems or dysphasia.

Lopatina notes that the phonetic side of speech is a close interaction of its main components: sound pronunciation and prosody (Lopatina and Loginova, 2005). Various phonetic means of formulating a statement (tempo, rhythm, stress, intonation) closely interact, defining both the semantic content and the speaker's attitude towards the content. In children with impaired dysarthria, prosodic disorders affect clarity, articulacy, and emotional speech pattern.

In the works of Mastyukova, devoted to the study of the speech of children with dysarthria, a violation of their speech tempo, as well as the difficulty of using dynamic, rhythmic and melodic stresses, is noted (Mastyukova and Ippolitova, 1985).

Dysarthria is a motor speech disorder characterised by impaired articulation and speech intelligibility.

Barbara Tomik and Roberto J. Guiloff, in their study, found that dysarthric disorders occur in children with amyotrophic lateral sclerosis (Tomik and Guiloff, 2010).

In the study of Belyakova and Romanchuk, dedicated to studying the features of the intonation side of speech in children attending schools for children with severe speech impairments, it is noted that at the age of 11-12 they have persistent disorders of prosody (Belyakova et al., 2017). The most difficult tests are the conversion of sentences into interrogative ones, expressive reading of sentences of different structures. These data indicate that spontaneous improvement of prosody with age is not observed.

Many researchers of the structure of a defect in the case of erased dysarthria indicate stable violations of the intonational expressiveness of speech, the processes of perception and reproduction of intonational structures of the sentence (Chirkina, 2005). In this case, the imitation of interrogative and narrative intonation is the most preserving difficulty.

Perception and self-reproduction of the intonation structure cause significant difficulties in children. Moreover, the process of auditory differentiation of intonational structures is more disturbed than the process of their independent implementation. The violation of the prosodic side of speech, which is a diagnostic criterion for 
the differentiation of erased dysarthria and dyslalia, is common to children.

Intonation-expressive colouring of speech of children with erased dysarthria is sharply reduced. The voice suffers: it is either quiet or excessively loud; modulations in voice height and strength are not successful (a child cannot imitate sounds in a high and low voice, imitating the voice of animals). The timbre of speech is broken, and sometimes a nasal shade appears. The pace of speech is often accelerated. In some children, against the background of the chest register, there occurs falsetto, inhalation with aspiration, with raising shoulders; mainly upper thoracic (upper clavicular) respiration is noted; speech exhalation is weakened. In some children, speech exhalation is shortened, and they speak while inhaling. In this case, speech becomes cluttering. The children's speech is inexpressive; the diction is not clear. According to Arkhipova, when reciting a poem, the speech of the child is monotonous, gradually becoming less legible, the voice fades. Violations of the formation of the intonation structure of a sentence are observed, while the process of auditory differentiation of intonation structures is more disturbed than the process of their independent implementation (Arkhipova, 2006). The study of various prosodic components specially conducted by Arkhipova shows that, in the first place, perception and auditory differentiation of intonation structures is disturbed (Arkhipova, 2006). Many children with erased dysarthria have difficulties in perceiving the rhythm of isolated strokes, accented strokes. Reproduction of rhythms also causes difficulties. Tasks on the perception and reproduction of intonations are difficult for most children with erased dysarthria, active adult assistance is required. Children who make mistakes in perceiving and reproducing intonations do not notice their mistakes. They fail to perform the tasks for changing intonations (joy, sadness) on the material of one and the same phrase. In some cases, tasks cannot be performed when examining logical stress. Children with erased dysarthria cannot accentuate a particular word in a sentence.
When comprehending phrases with exaggerated logical stress, children often do not emphasise the accented word. When examining voice modulations in height and strength, some difficulties are also noted. It is not possible to intonate melodies (on vowels) from bottom to top and from top to bottom. In some cases, the strength of the voice cannot be changed. In general, the range of voice in children with erased dysarthria is narrowed (within 3-4 tones). The cause of voice disturbance in the case of erased dysarthria lies in the pathology of the efferent and afferent links of intonation control. Due to paresis, a particular limitation of the arbitrary movements of vocal folds of diaphragm muscles, disturbances relating to the efferent link appear. Afferent pathology is manifested in the insufficiency of the kinaesthetic analysis, in the violation of proprioceptive impulse from phonation and respiration organs.

In many studies, the prosodic side of speech of children with erased dysarthria is assessed as emotionally inexpressive and monotonous. Speech intelligibility decreases markedly with increasing speech load. The children's voice is evaluated by the following characteristics: weak, tuneless, deaf, hoarse, monotonous, tight, faint, tense, intermittent, nasalised and weakly modulated voice. These symptoms can be presented in the case of erased dysarthria in various combinations and varying degrees of severity. Nasalisation (in its pure form), that is, not caused by adenoids, is a rather rare symptom in the case of erased dysarthria. The research by Artemova revealed a relationship between the degree of development of the prosodic formation of speech utterance and the degree of formation of auditory self-control operations (Artemova, 2008). Artemova used the adapted methodology of Paan to identify the children's ability to highlight errors in their own speech. This technique allowed the author to determine the nature of prosodic disorders in preschool children: sensory, motor, and mixed.

Artemova identified 4 degrees of formation of the prosodic side of speech in preschoolers (with erased dysarthria, general speech underdevelopment, phonetic-phonemic speech 
underdevelopment, phonetic speech disorders) (Artemova, 2008).

Degree 1 (low) - gross violations of prosodic components. The disadvantages of the timbre, strength, and pitch of the voice are pronounced, visible to a child and people around him/her. Degree 2 (insufficient) - voice changes are insignificant. Changes in prosody concern some or all of its components. Degree 3 (medium) - an intermittent or unstable deviation from the standard by one or more prosodic characteristics. Spontaneous speech is quite intonated, but when performing special tasks, inaccuracies, or individual errors in the reproduction of rhythmic and melodic patterns are possible.

Degree 4 (high). The formation of all prosodic characteristics. Children have a normal timbre, the range of their voice in strength and height corresponds to age norms. The tempo-rhythmic side of speech is formed.

Studies confirm the heterogeneity and variability of prosodic disorders in preschool children with erased dysarthria, with general speech underdevelopment and phonetic-phonemic impairment. At the same time, a correlation was found between the prosodic side of speech and the severity of a speech defect.

Breathing is one of the most critical body functions, providing a continuous supply of oxygen to all organs and tissues.

Nancy Solomon, in her works, examined the agerelated dynamics of the development of speech breathing (Nancy Solomon Speech Breathing, 1998). In her work, she studied the features of speech breathing in children with infantile cerebral palsy. Children with infantile cerebral palsy necessarily have speech disorders in the form of dysarthria.

A respiratory function is related to the regulation of other functional systems, and primarily - to the activity of the cardiovascular system. Changes in the rhythm and respiratory rate directly affect the change in heart rate. The socalled respiratory sinus arrhythmia (RSA) is manifested in the fact that normally during inspiration the heart rate increases and during exhalation it decreases.
If the rhythms of respiration and cardiac activity do not substantially coincide, the rate of respiratory sinus arrhythmia (RSA) is significantly reduced, which, according to Smetankin, indicates an additional strain on the heart (Smetankin, 2007). A prolonged state of overstraining inevitably leads to a breakdown of adaptation mechanisms and the development of somatic pathology.

If the respiratory and cardiac rhythms are relatively synchronised, well-marked stable RSA is maintained; therefore, the cardiovascular system operates in the optimal mode of strain distribution.

The authors' experiment was aimed at the formation of speech breathing in preschool children with erased dysarthria using the biological feedback method.

The hardware-software complex "BF" helps transmit the information to a person by using technical means about how his/her organs and systems function (in the form of graphs and game plots), which display the heart rate, respiration rate, RSA (respiratory sinus arrhythmia) (BOS hardware and software systems).

This computer program, combined with a set of standard and variable games, turns the logotherapeutic procedure into an entertaining game, which corresponds to neoplasms of preschool age and the leading type of children's activity.

"Logo" mode. This type of session is designed to work with speech therapy disorders. The first stage of the work is the setting of diaphragmatic relaxation breathing in the "Cardio" mode (at least 6 sessions). Further, the work is carried out in a special "Logo" mode containing the stimulus material for staging a continuous speech reproduction during expiration. The course consists of a minimum of $15-18$ sessions of $20-30$ minutes.

\section{The Organisation of the Study}

At the initial stage of this study, the authors carried out a stating experiment by using the hardware-diagnostic complex "BF" to identify the level of formation of diaphragmatic 
relaxation breathing in preschool children with an erased form of dysarthria.

Then the content of the formative experiment was developed using the hardware-diagnostic complex "BF" (BF-Health Room, Logotherapeutic Room) and a set of exercises proposed by Belyakova for the formation of speech breathing in preschool children.

The developmental session began with a set of exercises proposed by Belyakova for the formation of speech breathing in preschool children (a light toy is placed on the stomach of a child in a lying position on the diaphragm. A child in a lying position puts his/her palm on the diaphragm. A child in a sitting position puts his/her palm on the diaphragm) (Belyakova et al., 2017). These exercises were used to form the child's ability to control work both visually and tactilely, so that the child felt and understood how to "breathe with the abdomen", i.e. with the diaphragm.

Further, to consolidate the skill and the ability to control correct diaphragmatic relaxation breathing, the children studied on the hardwaresoftware complex "BF". During one lesson, the children were asked to go through 4 periods -2 periods of "rest", where a child breathed following his/her characteristic breathing pattern and 2 periods of "work", where a child had to breathe in accordance with the given instructions for developing diaphragmatic relaxation breathing:

Take a light, short breath through your nose without straining and holding your breath and, at the same time, inflate your stomach like a balloon, then smoothly switch to a uniform continuous exhalation through your mouth slightly open, while blowing your stomach like a balloon, returning it to the initial position. The exhalation is at least 2 times longer than the inspiration; there should be no breath-holding.

The game plots and graphs showed the child how correctly he/she could breathe. The duration of each period was 3 minutes (BOS hardware and software systems, 2019).

At the end of the lesson, the quantitative information was displayed on the screen about what level of RSA the child had in the "rest" and "work" modes, how many respiratory movements the child made during a certain period of work on the hardware-diagnostic complex "BF".

Further, the results of the study were qualitatively processed. To confirm the research hypothesis, the authors used the method of mathematical statistics (Student's t-test).

\section{Results and Discussion}

The results of the formative experiment showed that $60 \%$ of the children in the study sample did not have diaphragmatic breathing, among them clavicular breathing prevailed; $38 \%$ of the children in the study sample, if given instructions, used diaphragmatic breathing, but in everyday life, they usually had supercostal breathing. Only $2 \%$ of children with erased dysarthria had diaphragmatic breathing. This situation reflects a classical picture of the development of the prosodic side of speech in the case of an erased form of dysarthria.

In the process of conducting the formative experiment, a slight upward trend in the indices of the formation of the skill of diaphragmatic relaxation breathing can be seen, especially in the first seven sessions. Thus, by the eighth session, $54 \%$ of children in the process of working on the hardware-diagnostic complex "BF-Health" had an increase in RSA (respiratory sinus arrhythmia), which indicates the formation of the skill of diaphragmatic relaxation breathing both in the "rest" and "work" modes. In $28 \%$ of children, the skill of diaphragmatic relaxation breathing is only in the "work" mode, that is, only when a child sees directly on the screen how he/she can breathe with the diaphragm.

As our observations show, if a child is interested in conducting a lesson, then he listens carefully to the instruction and tries to follow it throughout the entire correctional-developing lesson. Thus, motivation plays an important role in achieving high results during the child's classes. Some children's skill in diaphragmatic relaxation breathing develops more difficultly. In the authors' opinion, this is due to the individual personality characteristics of a child. In particular, Yaroslav showed by his behaviour that he did not accept the task assigned to him 
by a speech therapist, hardly made contact during the session, even though the examiners tried to interest him, including a variety of game plots in the computer program. At the sessions, Yaroslav was closed up and uncommunicative, more self-involved, did not show any emotional response in relation to the results of the task performance. A teacher-speech therapist at a kindergarten, who works directly with this group of children, noted in an individual conversation that Yaroslav behaves in the same way at other speech therapy sessions. If only the session is very interesting to him, a child will show good results.

Children with an attention-deficit hyperactivity syndrome (34\% of the total sample) had difficulty in forming a skill of diaphragmatic relaxation breathing. Such children have spasmodic indicators of the development of a skill of diaphragmatic relaxation breathing (speech breathing). For example, Timur's RSA indicator at one of the sessions was 18 (which indicates an average degree of formation of diaphragmatic breathing), and the next day the RSA indicator decreased to 8 (this is a low level of diaphragmatic breathing). This is a significant decrease. There were several such hikes during the work.

Almost all children with an erased form of dysarthria, who attended developmental sessions on the formation of speech breathing, at the initial stage of work raised their shoulders while breathing. At the same time, the diaphragm was not involved in the act of breathing. There was also an aspirated breath.

Therefore, the authors also conducted breathing exercises among children, along with the use of the hardware-diagnostic complex "BF". The use of respiratory gymnastics since the second developmental session has shown an improvement in the formation of diaphragmatic relaxation breathing skills. Thus, the children in accordance with the instructions tried to breathe, activating the diaphragmatic type of breathing. At the same time, they could see on the computer screen whether they were following the instructions correctly, how much their breathing corresponded to the diaphragmatic type of breathing. Working with children suffering from dysarthria on the formation of speech breathing should be systematic and planned. As has already been noted, many children with erased dysarthria have the impaired prosodic side of speech. They have falsetto, breath with aspiration, with raising of shoulders; mainly upper thoracic (upper clavicular) respiration is noted; weakened speech exhalation. Some children have shortened speech exhalation, and they speak while inhaling - in this case, speech becomes cluttering.

According to the results of corrective sessions, $37 \%$ of the children included in the study sample have formed a skill of diaphragmatic breathing. In $43 \%$ of children, the skill of diaphragmatic relaxation breathing is not stable; it must be worked out and controlled, in particular, using the method of biological feedback. In $20 \%$ of the children, the skill of diaphragmatic relaxation breathing has not been formed. This group mainly includes children with an attention deficit hyperactivity syndrome.

A statistical analysis of the results of ascertaining and formative experiments using Student's t-test showed $(t=3.15$ at $p \leq 0.01)$ that there were differences in the indices of formation of diaphragmatic relaxation breathing skills at the beginning and the end of a series of correctional developmental sessions. Therefore, the method of biological feedback contributes to the formation of the skill of diaphragmatic relaxation breathing in preschool children with an erased form of dysarthria. However, in hyperactive children, the process of formation of speech breathing takes a longer time.

\section{Conclusion}

The principal aim of this study was to examine the formation of speech breathing in preschool children with erased dysarthria using the biological feedback method in speech therapy.

The hardware-diagnostic complex "BF" promotes the formation of diaphragmatic speech breathing in children with an erased form of dysarthria. 
However, for children with an erased form of dysarthria, for a better effect, longer work is required for the formation of speech breathing than 20 correctional developmental sessions.

When conducting speech therapy sessions for children with speech disorders, it is vital to take into account individual personality characteristics. In children suffering from erased dysarthria and with an attention deficit hyperactivity syndrome, the process of formation of diaphragmatic relaxation breathing is more complicated.

Because speech breathing is formed only by the age of 10 and it begins to correspond to the syntagmatic division of texts, it is vital to prevent the violation of speech breathing in children with an erased form of dysarthria even in preschool age until the incorrect stereotype of speech breathing has finally fixed.

Following the objectives of this study, the authors proposed the following recommendations for the formation of speech breathing in preschool children with an erased form of dysarthria:

- The work on the formation of speech breathing should be staged, systematic, and long-lasting.

- It is crucial for children with an erased form of dysarthria to include exercises on the formation of speech breathing in daily speech therapy sessions.

- When conducting correctional developmental sessions for the formation of speech breathing, it is necessary to use a variety of methods and technologies including both a series of physical exercises (breathing exercises) and innovative techniques (hardware-diagnostic complexes) so that children do not overwork and hold interest in performing tasks throughout the session. This is especially important for children suffering from dysarthria, as they are easily distracted; their attention is not stable.

- In the formation of speech breathing in children with an erased form of dysarthria, it is necessary to use graphic images, game plots, and motor exercises to quickly form speech breathing skills and activate all analyser systems.

- When conducting correctional developmental sessions among children with speech disorders, it is essential to take into account individual personality traits.

\section{References}

Arkhipova, E.F. (2006). Erased dysarthria in children. Moscow: AST: Astrel, 2006, 344 p.

Artemova, E.E. (2008). Features of the formation of prosodics in preschool children with speech disorders. Monograph. Moscow: MGGU named after M.A. Sholokhov, 123 p.

Belyakova, L.I., Goncharova, N.N., Filatova, Yu.B., \& Khatnyukova-Shishkova T.G. (2017). Health-saving technologies for the development of speech in children. Moscow: National Book Center, $80 \mathrm{p}$.

Bernardi, A. (2019). The capability approach and organizational climate as tools to study occupational health and safety, Insights into Regional Development 1(2): 155-

169. https://doi.org/10.9770/ird.2019.1.2(6)

BOS hardware and software systems. Retrieved on 04 May 2020 from, http://www.biosvyaz.com

Blumenfeld, H. (2017). Considering Dysarthria: A Speech Disorder 'On the Margins' Biology 202 Retrieved on 04 May 2020 from, https://serendipstudio.org/exchange/serendipu pdate/considering-dysarthria-speech-disordermargins

Brigas, C. (2019). Modeling and Simulation in an Educational Context: Teaching and Learning Sciences. Research in Social Sciences and Technology, 4(2), 1-12. Retrieved on 04 May 2020 from, https://ressat.org/index.php/ressat/article/vie $\mathrm{w} / 418$

Chirkina G.V. (2005). Fundamentals of speech therapy work with children. Ed. Moscow: ARKTI, 2005. 412 p. 
Chistobaev A.I., Semenova A.Z., \& Grudtsyn N.A. (2018). Dynamics and strategic directions of public health preservation in Russian Federation. Entrepreneurship and Sustainability Issues 6(3): 1180-1192.

http://doi.org/10.9770/jesi.2019.6.3(23)

Darley, F. L., Aronson, A. E., \& Brown, J. R. (1969). Clusters of deviant speech dimensions in the dysarthrias. Journal of Speech and Hearing Research, 12(3), 462-496.

Dinh, H. (2019). Towards a Knowledge-rich Curriculum. Journal Of Curriculum Studies Research, 1(1), 54-70. Retrieved on 04 May 2020 from, https://curriculumstudies.org/index.php/CS/arti cle/view/6

Etherington, M. (2019). The Challenge with Educational Transformation. Journal of Culture and Values in Education, 2(1), 96-112. Retrieved on 04 May 2020 from,

http://cultureandvalues.org/index.php/JCV/arti cle/view/33 [Date of access 04.05.2020]

Hitos, S. F., Arakaki, R., Solé, D., \& Weckx, L. L. M. (2013). Oral breathing and speech disorders in children. Jornal De Pediatria, 89(4), 361-365. doi:10.1016/j.jped.2012.12.007

Lopatina, L.V., \& Loginova, E.A. (2005). Speech therapy work with preschool children with minimal dysarthria disorders: a Training manual. Saint Petersburg: Soyuz Publishing House. 192p.

Kireev, B., Zhundibayeva, A., \& Aktanova, A. (2019). Distance learning at higher education institutions: Results of an experiment. Journal of Social Studies Education Research, 10(3), p. 387-403.

Mastyukova, E.M., \& Ippolitova, M.V. (1985). Speech disturbance in children with cerebral palsy: Prince for a speech therapist. Moscow: Education, $13 \mathrm{p}$.

Nancy Solomon Speech Breathing in AbleBodied Children and Children with Cerebral Palsy. American Journal of Speech-Language Pathology 7(2), p. 61. May 1998.
Pavlyshyn, L., Voronkova, O., Yakutina, M., \& Tesleva, E. (2019). Ethical problems concerning dialectic interaction of culture and civilization. Journal of Social Studies Education Research, 10(3), p. 236-248.

Rupeika-Apoga, R., Romanova, I., Bule, L. \& Thalassinos, Y. (2019). The Impact of Population Ageing and Social Stratification: The Case of Latvia. International Journal of Economics \& Business Administration, 7(1), p. 49-63.

Russell, J. (1999). Love Childhood Motor Speech Disability (2nd Edition). Pearson; 2 edition (October 21, 1999). p. 202

Saenko, N., Voronkova, O., Volk, M., \& Voroshilova, O. (2019). The social responsibility of a scientist: Philosophical aspect of contemporary discussions. Journal of Social Studies Education Research, 10(3), p. 332-345.

Shrestha, M. (2019). Influences of gender and locale on teachers' job satisfaction. Research in Educational Policy and Management, 1(1), 1732. Retrieved on 04 May 2020 from, https://repamjournal.org/index.php/REPAM/ar ticle/view/7

Solomon, N.P., Charron S. (1998). Speech Breathing in Able-Bodied Children and Children With Cerebral Palsy: A Review of the Literature and Implications for Clinical Intervention.

American Journal of Speech-Language Pathology, 7, p. 61-78.

Smetankin, A.A. (2007). Health for $5+$ : a book for everyone: for children and adults, for dads and mothers, for grandparents. Saint Petersburg: Biocommunication, $160 \mathrm{p}$.

Tarman, B. (2016). Innovation and Education. Research in Social Sciences and Technology, 1(1). Retrieved on 04 May 2020 from, http://ressat.org/index.php/ressat/article/view /3

Tomik, B., \& Guiloff, R. J. (2010). Dysarthria in amyotrophic lateral sclerosis: A review. Amyotrophic Lateral Sclerosis. 11, p. 4-15. Volkova, L.S. (2004). Speech Therapy. Moscow, $368 \mathrm{p}$. 